\title{
Do you believe that processed EEG helps to prevent intraoperative awareness?
}

\author{
Dong Woo Han \\ Department of Anesthesiology and Pain Medicine, Anesthesia and Pain Research Institute, Gangnam Severance \\ Hospital, Yonsei University College of Medicine, Seoul, Korea
}

This month's edition of the Korean Journal of Anesthesiology contains a paper focusing on the comparison between cerebral state index (CSI) and bispectral index (BIS) during desflurane anesthesia in which Cho et al. [1] reported that CSI can be used as a good substitute for BIS, regardless of the kind of anesthetic used.

Brain function monitors for assessing the depth of anesthesia or hypnosis have been commercially available since the 1990s. The processed electroencephalogram (pEEG) has been used to monitor the depth of anesthesia by numerical representation. Many available anesthetic depth monitors including the BIS monitor, M-Entropy, Narcotrend monitor, cerebral function monitor, CSI, patient state index, and lower esophageal contractility monitor have been introduced, and studied extensively for clinical utility and performance. Initial studies suggested that the use of pEEG technology could significantly decrease the incidence of unintended intraoperative awareness events during general anesthesia. However, recently published trials examining the use of the pEEG, such as BIS during general anesthesia using inhaled anesthetic agent, have not been able to show superiority over the technique of maintaining an end-tidal anesthetic-agent concentration of $>0.7$ age-adjusted minimum alveolar con-

Corresponding author: Dong Woo Han, M.D., Ph.D.

Department of Anesthesiology and Pain Medicine, Anesthesia and Pain Research Institute, Gangnam Severance Hospital, Yonsei University College of Medicine, 211 Eonju-ro, Gangnam-gu, Seoul 06273, Korea

Tel: 82-2-2019-6891, Fax: 82-2-3463-0940

Email: hanesth@yuhs.ac

ORCID: https://orcid.org/0000-0002-8757-663X

Received: October 31, 2018.

Accepted: November 1, 2018.

Korean J Anesthesiol 2018 December 71(6): 427-429

https://doi.org/10.4097/kja.d.18.00309 centration (MAC) [2]. Recently, neither careful monitoring of a patient to reduce the frequency of intraoperative awareness, nor depth monitoring of anesthesia, are routinely indicated in patients undergoing procedures under general anesthesia. The ASA's Practice Advisory for intraoperative awareness recommended that the decision on whether to use a brain function monitor should be made on the basis of a case-by-case by tailoring each patient's anesthetic protocol based on one's comorbidities and the procedure performed [3]. Selective use of pEEG may be useful for the prevention of intraoperative awareness during the administration of total intravenous anesthesia (TIVA) combined with neuromuscular blockade, and for avoiding drug overdose in the elderly. The incidence of intraoperative awareness with explicit recall is two-fold to four-fold higher when using the TIVA technique with propofol, when compared to the use of a volatile anesthetic. In addition, the use of a neuromuscular blockade drug can dramatically increase the risk of awareness while pEEG monitoring significantly reduces that risk [4]. The intraoperative use of pEEG can be recommended for the elderly patient to minimize postoperative delirium by avoiding deep levels of anesthesia that may result in unnecessary burst suppression [5]. Several studies show that exposure to deep anesthesia is associated with the occurrence of delirium, especially in vulnerable patients, such as the elderly [6,7]; but all results have not been consistent [8].

Studies have found that BIS values correlated with outcomes in other ways. The 'triple low' (the combination of intraoperative mean arterial pressure $<75 \mathrm{mmHg}$, BIS $<45$, and $\mathrm{MAC}<$ 0.8 ) is associated with an increased risk of perioperative mortality, particularly if the 'triple low' lasts for more than $15 \mathrm{~min}$ $[9,10]$. Another use is that, the pEEG can predict the outcome of cardiac arrest and traumatic brain injury. Mean BIS values in the first $24 \mathrm{~h}$ after resuscitation are higher in patients with good outcomes, when compared with those with poor outcomes [11]; and patients with high BIS values (> 60) after traumatic brain

(c) This is an open-access article distributed under the terms of the Creative Commons Attribution Non-Commercial License (http://creativecommons.org/ licenses/by-nc/4.0/), which permits unrestricted non-commercial use, distribution, and reproduction in any medium, provided the original work is properly cited. 
injury have a high survival rate and Glasgow Coma Score [12]. BIS monitors can also be used to guide burst suppression therapy (burst suppression ratio of 15-30) applied during deep coma for the treatment of increased intracranial pressure. Intensive care unit (ICU), pEEG monitoring in patients receiving continuous infusions of sedatives and neuromuscular blockade can give both economic and personal benefits, even though BIS variability is relatively high in ICU patients. Using pEEG for titrating the dosage of sedating agents can also decrease the risk of medical complications associated with over-sedation, such as hypotension, ventilator-associated pneumonia, and longer ICU stays, with a secondary benefit of financial savings from reduced drug doses and decreased time to tracheal extubation [13,14]. Some studies have shown that a decrease in BIS value may correlate with cerebral ischemia, suggesting that pEEG is a useful tool in detecting cerebral ischemia or injury $[15,16]$. Others, however, have failed to demonstrate an association $[17,18]$, which could be partly explained by the inability of pEEG to assess subcortical regions of the brain. Therefore, current literature cannot support pEEG monitoring as a sensitive modality for detecting cerebral ischemia, whether the monitor uses unilateral or bilateral EEG channels.

For more accurate interpretation of the pEEG, clinicians should recognize its several limitations. Patients' pathophysiologic states (e.g., Hypothermia, hypoglycemia, acid-base abnormalities and pre-existing neurologic disorder), degree of muscle activity, and muscle relaxants can affect the pEEG indices. Other limitations include undetected artifacts (e.g., electrocautery, vibration from forced air warmers) and time lag for the index to display after the occurrence of an event that may affect the pEEG [19]. When there are changes in the pEEG, the clinicians might be able to determine from the raw EEG trace, whether the change is caused by an artifact or by true changes in the hypnotic state. It should also be noted that changes occur in the raw EEG trace about one minute before they are reflected in the pEEG indices.

\section{References}

1. Cho S, Kim S, Hyun D, Yoon H, Han JW, Kim JS. Comparison between cerebral state index and bispectral index during desflurane anesthesia. Korean J Anesthesiol 2018; 71: 447-52.

2. Avidan MS, Jacobsohn E, Glick D, Burnside BA, Zhang L, Villafranca A, et al. Prevention of intraoperative awareness in a high-risk surgical population. N Engl J Med 2011; 365: 591-600.

3. American Society of Anesthesiologists Task Force on Intraoperative Awareness. Practice advisory for intraoperative awareness and brain function monitoring: a report by the american society of anesthesiologists task force on intraoperative awareness. Anesthesiology 2006; 104: 847-64.

4. Checketts MR, Alladi R, Ferguson K, Gemmell L, Handy JM, Klein AA, et al. Recommendations for standards of monitoring during anaesthesia and recovery 2015: Association of Anaesthetists of Great Britain and Ireland. Anaesthesia 2016; 71: 85-93.

5. Aldecoa C, Bettelli G, Bilotta F, Sanders RD, Audisio R, Borozdina A, et al. European Society of Anaesthesiology evidence-based and consensus-based guideline on postoperative delirium. Eur J Anaesthesiol 2017; 34: 192-214.

6. Fritz BA, Kalarickal PL, Maybrier HR, Muench MR, Dearth D, Chen Y, et al. Intraoperative Electroencephalogram Suppression Predicts Postoperative Delirium. Anesth Analg 2016; 122: 234-42.

7. Hernandez BA, Lindroth H, Rowley P, Boncyk C, Raz A, Gaskell A, et al. Post-anaesthesia care unit delirium: incidence, risk factors and associated adverse outcomes. Br J Anaesth 2017; 119: 288-90.

8. Whitlock EL, Torres BA, Lin N, Helsten DL, Nadelson MR, Mashour GA, et al. Postoperative delirium in a substudy of cardiothoracic surgical patients in the BAG-RECALL clinical trial. Anesth Analg 2014; 118: 809-17.

9. Sessler DI, Sigl JC, Kelley SD, Chamoun NG, Manberg PJ, Saager L, et al. Hospital stay and mortality are increased in patients having a "triple low" of low blood pressure, low bispectral index, and low minimum alveolar concentration of volatile anesthesia. Anesthesiology 2012; 116: 1195-203.

10. Leslie K, Myles PS, Forbes A, Chan MT. The effect of bispectral index monitoring on long-term survival in the B-aware trial. Anesth Analg 2010; 110: 816-22.

11. Stammet P, Collignon O, Werer C, Sertznig C, Devaux Y. Bispectral index to predict neurological outcome early after cardiac arrest. Resuscitation 2014; 85: 1674-80.

12. Dunham CM, Ransom KJ, McAuley CE, Gruber BS, Mangalat D, Flowers LL. Severe brain injury ICU outcomes are associated with Cranial-Arterial Pressure Index and noninvasive Bispectral Index and transcranial oxygen saturation: a prospective, preliminary study. Crit Care 2006; 10: R159.

13. Consales G, Chelazzi C, Rinaldi S, De Gaudio AR. Bispectral Index compared to Ramsay score for sedation monitoring in intensive care units. Minerva Anestesiol 2006; 72: 329-36.

14. Triltsch AE, Welte M, von Homeyer P, Grosse J, Genähr A, Moshirzadeh M, et al. Bispectral index-guided sedation with dexmedetomidine in intensive care: a prospective, randomized, double blind, placebo-controlled phase II study. Crit Care Med 2002; 30: 1007-14. 
15. Estruch-Pérez MJ, Ausina-Aguilar A, Barberá-Alacreu M, Sánchez-Morillo J, Solaz-Roldán C, Morales-Suárez-Varela MM. Bispectral index changes in carotid surgery. Ann Vasc Surg 2010; 24: 393-9.

16. Estruch-Pérez MJ, Barberá-Alacreu M, Ausina-Aguilar A, Soliveres-Ripoll J, Solaz-Roldán C, Morales-Suárez-Varela MM. Bispectral index variations in patients with neurological deficits during awake carotid endarterectomy. Eur J Anaesthesiol 2010; 27: 359-63.

17. Bonhomme V, Desiron Q, Lemineur T, Brichant JF, Dewandre PY, Hans P. Bispectral index profile during carotid cross clamping. J Neurosurg Anesthesiol 2007; 19: 49-55.

18. Deogaonkar A, Vivar R, Bullock RE, Price K, Chambers I, Mendelow AD. Bispectral index monitoring may not reliably indicate cerebral ischaemia during awake carotid endarterectomy. Br J Anaesth 2005; 94: 800-4.

19. Fahy BG, Chau DF. The technology of processed electroencephalogram monitoringdevices for assessment of depth of anesthesia. Anesth Analg 2018; 126: 111-7. 